\title{
The 2q37-deletion syndrome: an update of the clinical spectrum including overweight, brachydactyly and behavioural features in 14 new patients
}

\author{
Camille Leroy ${ }^{1,2,3}$, Emilie Landais ${ }^{1,2,4}$, Sylvain Briault ${ }^{5}$, Albert David ${ }^{6}$, Olivier Tassy ${ }^{7}$, Nicolas Gruchy ${ }^{8}$, \\ Bruno Delobel $^{9}$, Marie-José Grégoire ${ }^{10}$, Bruno Leheup ${ }^{3,11}$, Laurence Taine ${ }^{12}$, Didier Lacombe ${ }^{12}$, Marie-Ange \\ Delrue $^{12}$, Annick Toutain ${ }^{13}$, Agathe Paubel ${ }^{13}$, Francine Mugneret ${ }^{14}$, Christel Thauvin-Robinet ${ }^{3,15}$, \\ Stéphanie Arpin ${ }^{13}$, Cedric Le Caignec ${ }^{6}$, Philippe Jonveaux ${ }^{3,10}$, Mylène Beri ${ }^{10}$, Nathalie Leporrier ${ }^{8}$, \\ Jacques Motte $^{16}$, Caroline Fiquet ${ }^{17,18}$, Olivier Brichet ${ }^{16}$, Monique Mozelle-Nivoix ${ }^{1,3}$, Pascal Sabouraud ${ }^{16}$, \\ Nathalie Golovkine ${ }^{19}$, Nathalie Bednarek ${ }^{20}$, Dominique Gaillard ${ }^{1,2,3}$ and Martine Doco-Fenzy ${ }^{\star, 1,2,3,18}$
}

The $2 q 37$ locus is one of the most commonly deleted subtelomeric regions. Such a deletion has been identified in $>100$ patients by telomeric fluorescence in situ hybridization (FISH) analysis and, less frequently, by array-based comparative genomic hybridization (array-CGH). A recognizable '2q37-deletion syndrome' or Albright's hereditary osteodystrophy-like syndrome has been previously described. To better map the deletion and further refine this deletional syndrome, we formed a collaboration with the Association of French Language Cytogeneticists to collect 14 new intellectually deficient patients with a distal or interstitial 2q37 deletion characterized by FISH and array-CGH. Patients exhibited facial dysmorphism (13/14) and brachydactyly (10/14), associated with behavioural problems, autism or autism spectrum disorders of varying severity and overweight or obesity. The deletions in these 14 new patients measured from 2.6 to $8.8 \mathrm{Mb}$. Although the major role of HDAC4 has been demonstrated, the phenotypic involvement of several other genes in the deleted regions is unknown. We further refined the genotype-phenotype correlation for the 2q37 deletion. To do this, we examined the smallest overlapping deleted region for candidate genes for skeletal malformations (facial dysmorphism and brachydactyly), overweight, behavioural problems and seizures, using clinical data, a review of the literature, and the Manteia database. Among the candidate genes identified, we focus on the roles of PRLH, PER2, TWIST2, CAPN10, KIF1A, FARP2, D2HGDH and PDCD1.

European Journal of Human Genetics (2013) 21, 602-612; doi:10.1038/ejhg.2012.230; published online 17 October 2012

Keywords: 2q37-deletion syndrome; genotype-phenotype correlation; intellectual disability; overweight; brachydactyly; AHO-like syndrome

\section{INTRODUCTION}

Deletions of the distal 2q37 region involve the last cytogenetic band on the long arm of chromosome 2, which is divided into three subbands: 2 q37.1, 2q37.2 and 2q37.3. The last sub-band notably contains a small subtelomeric region, 2qtel, which displays non-deleterious polymorphic deletions or duplications. 2q37 Deletions have been described in $>115$ patients in the literature, associated clinically with intellectual deficiency, brachydactyly, obesity and short stature, ie, the so-called Albright's hereditary osteodystrophy-like (AHO-like) syndrome (OMIM: brachydactyly-mental retardation syndrome no.
600430; http://omim.org/). A precise mapping of the deleted regions is not often available, as most published cases have been characterized by conventional cytogenetics, subtelomeric fluorescence in situ hybridization (FISH) or microsatellite markers, and array-based comparative genomic hybridization (array-CGH) has only been used in a few studies. ${ }^{1-10}$

At least 197 genes are located in the $2 \mathrm{q} 37$ region $(230.7-243.2 \mathrm{Mb}$; Hg19; NCBI map viewer http://www.ncbi.nlm.nih.gov/mapview/). Of these, 11 have been reported as being potentially related to the $2 \mathrm{q} 37$ deletion phenotype so far, ${ }^{5,10-17}$ but the phenotypic implications of

${ }^{1} \mathrm{CHU}-$ Reims, HMB, Service de génétique, Reims, France; ${ }^{2}$ UFR de médecine, SFR-CAP SANTE, Université de Reims Champagne-Ardenne, Reims, France; ${ }^{3}$ Centre de Référence Maladies Rares « Anomalies du Développement et Syndromes Malformatifs » de la Région Est, France; ${ }^{4} \mathrm{CHU}-$ Reims, HMB, Plateforme Régionale de Biologie Innovante, Reims, France; ${ }^{5} \mathrm{CHR}$-Orléans, Service de génétique, Laboratoire d’Immunologie et Neurogénétique expérimentales et moléculaires, Hôpital de la Source, UMR 7355 , CNRS_Université d'Orléans, Orléans, France; ${ }^{6} \mathrm{CHU}-$ Nantes, Service de génétique médicale, Nantes, France; ${ }^{7}$ Institut de Génétique et de Biologie Moléculaire et Cellulaire (IGBMC)-IIlkirch, CNRS (UMR 7104), Inserm (U964), Université de Strasbourg, Illkirch, France; ${ }^{8}$ CHU-Caen, Département de génétique, Hôpital de la Côte de Nacre, Caen, France; ${ }^{9} \mathrm{CHU}$-Lille, Centre de génétique chromosomique, Hôpital Saint Vincent de Paul, Lille, France; ${ }^{10} \mathrm{CHU}-N a n c y$, Laboratoire de cytogénétique et génétique moléculaire, Hôpital Brabois, Université de Lorraine, Vandoeuvre-lès-Nancy, France; ${ }^{11} \mathrm{CHU}-N a n c y$, Service de médecine infantile 3 et de génétique clinique, Hôpital Brabois enfants, Université de Lorraine, Vandoeuvre-lès-Nancy, France; ${ }^{12} \mathrm{CHU}$-Bordeaux, Service de génétique médicale, Laboratoire MRGM, Hôpital Pellegrin, Université de Bordeaux, Bordeaux, France; ${ }^{13} \mathrm{CHRU}$-Tours, Service de génétique, Hôpital Bretonneau, Tours, France; ${ }^{14} \mathrm{CHU}$-Dijon, Laboratoire de cytogénétique, Plateau technique de Biologie, Dijon, France; ${ }^{15} \mathrm{CHU}$-Dijon, Centre de génétique, Hôpital du Bocage, Equipe d'accueil GAD, IFR 100 Santé STIC, Université de Bourgogne, Dijon, France; ${ }^{16} \mathrm{CHU}$-Reims, American Memorial Hospital, Service de Pédiatrie A, Reims, France; ${ }^{17} \mathrm{CHU}$-Reims, American Memorial Hospital, Service de Chirurgie pédiatrique, Reims, France; ${ }^{18}$ Université de Reims Champagne-Ardenne, Reims, France; ${ }^{19} \mathrm{CHU}$-Reims, Service de psychothérapie de l'enfant et l'adolescent, Hôpital Robert Debré, Reims, France; ${ }^{20} \mathrm{CHU-Reims,} \mathrm{American}$ Memorial Hospital, Service de Pédiatrie B, Reims, France

${ }^{*}$ Correspondence: Professor M Doco-Fenzy, CHU-Reims, HMB, Service de Génétique, 45 rue Cognacq Jay, EA3801, Reims 51092 , France. Tel: + 33326788582 ; Fax: + 333267841 45; E-mail: mdocofenzy@chu-reims.fr

Received 23 May 2012; revised 21 August 2012; accepted 11 September 2012; published online 17 October 2012 
most of them remain unknown. Among the candidate genes with a known phenotype, HDAC4 has notably been established as being responsible for brachymetaphalangy and intellectual disability. ${ }^{10}$ HDAC4 (MIM 605314) is a class II histone deacetylase that functions as a corepressor for DNA-binding transcription factors. It is a member of a group of enzymes that catalyses the removal of the acetyl group from lysine residues in histones and nonhistone proteins, resulting in the repression of transcription. They are ubiquitously expressed and have a role in transcriptional regulation, cell cycle progression and developmental events. HDAC4 is critical for proper skeletogenesis and chondrogenesis as well as neuronal survival. ${ }^{10}$

To obtain optimal genotype-phenotype correlations for other candidate genes, a collaborative study was initiated within the telomere network of the Association des Cytogénéticiens de Langue Française (Association of French Language Cytogeneticists, ACLF), enabling us to investigate 14 patients with a $2 \mathrm{q} 37$ deletion. The proximal breakpoint of the deletions, the parental origin of the deleted allele and the presence of the 2qtel polymorphism were analysed. The genotype-phenotype correlation analysis was then focused on some of the clinical features often associated with the 2q37 deletion, such as facial dysmorphism, brachydactyly, obesity, intellectual disability, seizures and behavioural disorders of the autistic spectrum (ASD). We used the data mining tool Manteia (http:// manteia.igbmc.fr/), and notably the rodent database (Mouse Genome Informatics (MGI), to optimize the search for candidate genes in a region of nearly $9 \mathrm{Mb}$ corresponding to our longest deletion (P1) from $2 \mathrm{q} 37.1$ to the telomere. This tool enabled us to propose a map of genes of interest for the main clinical features.

\section{MATERIALS AND METHODS}

\section{Population}

A collaborative study was set up to collect patients from seven centres for genetics (2002-2011). Informed consent was obtained for all patients tested. We recruited 14 patients ( 6 males and 8 females) with a known $2 \mathrm{q} 37$ deletion. Their ages ranged from 4 to 39 years. In total, 12 patients had terminal deletions, including 9 with purely distal deletions (P1-4, P6, P7, P10, P11 and $\mathrm{P} 13)$ and 3 with a deletion due to translocation (P8, P9 and P12). One patient had an interstitial $2 \mathrm{q} 37.2$ deletion (P5) and one patient had a $2 \mathrm{q} 37 \mathrm{del} / \mathrm{dup}$ rearrangement (P14).

\section{Methods}

All samples were obtained and analysis performed with the required consent from patients or their parents.

\section{Karyotyping}

Conventional cytogenetic analyses were performed on peripheral blood lymphocytes using the 550-band and/or 850-band level, including GTG and RHG banding for family members.

\section{Fluorescence in situ hybridization}

Academic and commercial probes were used. Among them RP11-341N2, GS$1011 \mathrm{O} 17$ and RP11-789L24 were used for diagnosis and to test the 2qtel polymorphism in parents and probes RP11-574K22 (P10), RP11-35I12 (P4) and RP11-332L11 (P4) for 2q37-deletion mapping. DNA for noncommercial probes was extracted from BAC and PAC colonies (N Carter, Sanger institute, Cambridge, UK), amplified with the illustra TempliPhi amplification kit (GE Healthcare, Piscataway, NJ, USA) and labelled with Cyanine3-dCTP or BiotindNTP using a nick translation method (BioNickTM DNA Labeling System, Invitrogen, Carlsbad, CA, USA). Hybridizations with commercial subtelomeric probes were performed according to the manufacturers' recommendations. Deletions were detected or confirmed by FISH in all 14 cases.

\section{Array-CGH}

Genomic DNA samples were extracted from peripheral blood using the QIAamp DNA Blood Midi kit (Qiagen, Valencia, CA, USA). Array-CGH was performed in all patients using the Agilent $60 \mathrm{k}$ (one patient) or $180 \mathrm{k}$ (nine patients) oligoarrays (Human Genome CGH Microarray Kit, Agilent Technologies, Santa Clara, CA, USA) or a BlueGnome (Cambridge, UK) BAC/PAC microarray (four patients). Random primer labelling and hybridization were carried out with sex-matched reference DNA according to the manufacturer's recommendations. Images were acquired using an Agilent or Axon scanner (Axon, Molecular Devices, Sunnyvale, CA, USA). Data were processed with Genepix 6.0 (Axon), BlueFuse (BlueGnome) or Feature Extraction (v9.5.3.1) software (Agilent Technologies), and results were analysed with the CGH Analytics software (v3.5.14; Agilent Technologies) using the ADM2 algorithm and a three-point filter.

\section{Quantitative PCR}

Quantitative PCR was performed in three patients (P8, P9 and P14) to clarify the position of the proximal breakpoint. Primers for the COL6A3 and HDAC4 genes were designed and tested using standard procedures (Eurogentec, Seraing, Belgium) on a LightCycler 480 Real-Time PCR System (Roche Diagnostics, Basel, Switzerland).

\section{Genotyping}

DNA genotyping of family members was performed by PCR amplification using standard procedures, with a panel of five sequence-tagged site microsatellite primers specific to chromosome 2 (region 2q37.3): D2S125, D2S2985, D2S2988, D2S2986 and D2S2585 (Eurogentec).

\section{Data-mining software manteia}

Manteia is a database integrating different kinds of data generated from human and animal models within a single framework, for in silico data mining. The system permits all experimental results and annotations from animal models to be linked to the human genome, making it possible to take advantage of data not available for humans to look for candidate genes responsible for genetic disorders. To highlight genes from the $2 \mathrm{q} 37$ deletion that could be responsible for each clinical feature observed in patients, we used Manteia to select those known to lead to obesity, abnormal behaviour or bone abnormalities in humans or mice. The MGI mouse data set were used because it contains numerous annotations including those from knockout experiments to provide genotype-phenotype correlations not available for humans.

\section{RESULTS}

\section{Clinical characteristics}

The clinical features of the patients are summarized in Tables 1 and 2 .

The skeletal phenotype in the 2q37-deletion syndrome is well known and characteristic. In this series, 13/14 patients had a specific facial dysmorphism and 10/14 displayed brachydactyly type E (brachymetacarpy and/or brachymetatarsy). The facial dysmorphism was remarkable for frontal bossing (5/13), uncombable hair with a low frontal hairline (6/13), thin eyelids (10/13), thin palpebral fissures (8/13), a small nose with a V-shaped tip (11/13), protruding cheekbones (5/13), a small mouth with thin lips (12/13), smooth philtrum (11/13), low-set ears (8/13) and a large chin (7/13; Figure 1). Bilateral AHO-like brachydactyly was present in 10/14 patients and detected as early as 4 years of age. Brachymetacarpy was present in $9 / 13$ patients, always present and more severe in the fourth digital ray. The fifth digital ray was also shortened in four patients, and both the third and fourth digital rays in one patient. Brachymetatarsy was present in 6/11 patients. The fourth and fifth digital rays were most often shortened compared with the second and third digital rays. Broad halluces and asymmetrical legs were present in six and two patients, respectively, (Figure 1, Tables 1 and 2). Three patients 


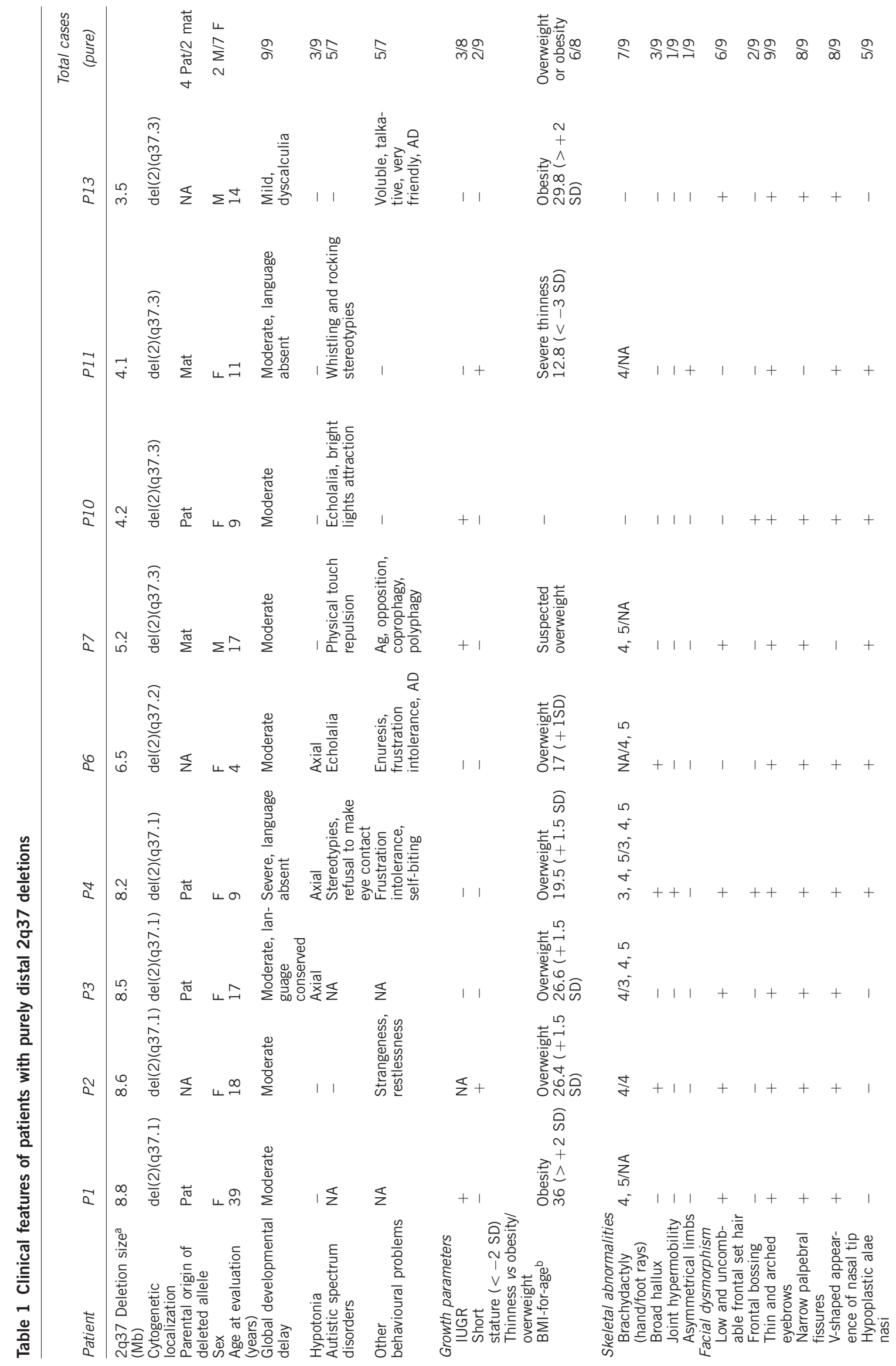


displayed short stature, $<2$ SD below the mean (P2, P8 and P11). Four patients had normal growth and stature (P9, P10, P12 and P14).

The body weight of the patients was evaluated and three categories defined: normal, overweight (a body mass index (BMI), higher than 1 SD above the mean) and obesity (BMI $>2$ SD), according to BMI-forage reference curves (WHO 2007). Five patients $>4$ years of age were overweight (P2, P3, P4, P6 and P8), and three patients $>7$ years of age were obese (P1, P5 and P13). For patient P7, the BMI was not available but clinical abdominal adiposity was described with a suspicion of overweight. One patient (P11) displayed delayed growth, with both BMI and height $<3$ SD below the mean.

Some patients had a past history of hypotonia (5/14). All patients had global developmental delays or mild to severe intellectual deficiencies according to their age. A lack of language acquisition was noted in two patients (9 years and 11 years). In total, 10 of 12 patients (not available for 2 patients) displayed abnormal behaviour, 7 displayed ASDs (7/12) and 4 displayed attention deficit or hyperactivity disorder (4/12). Seizures were present in 3/14 patients.

Malformations were also observed in 6 patients, including hernias (3/14), cryptorchidism (2/14), clubfeet (2/14) and cleft palate $(1 / 14)$.

\section{Deletion mapping}

Among the 14 patients, 13 (P1-4, P6-14) had distal 2q37 deletions ranging from 2.6 to $8.8 \mathrm{Mb}$. The largest encompassed 120 genes. The proximal breakpoints (Hg19) were distributed among the three subbands, 2q37.1, 2q37.2 and 2q37.3 (Table 3). Nine patients had purely distal deletions (P1-4, P6-7, P10-11 and P13). Three patients had a $2 \mathrm{q} 37$ deletion derived from translocations: $\operatorname{der}(2) \mathrm{t}(2 ; 6)(\mathrm{q} 37.3 ; \mathrm{p} 25.3)$ for P8, der(2)t(2;3)(q37.3;q29) for P9 and $\operatorname{der}(2) \mathrm{t}(2 ; 21)$ (q37.3;qter) for P12. P5 had an interstitial 2q37.2 deletion of $1.1 \mathrm{Mb}$ and P14 a $\operatorname{der}(2) \operatorname{dup}(2)(\mathrm{q} 37.2 \mathrm{q} 37.3) \operatorname{del}(2)(\mathrm{q} 37.3)$ rearrangement. The results of the mapping are summarized in Figure 2 and Table 3.

\section{Parental analysis}

We performed segregation analysis to correlate the phenotype with the origin of the $2 \mathrm{q} 37$-deleted chromosome (paternal or maternal) and to explore the hypothesis that the 2qtel polymorphism could be a predisposing deletion in the parents.

Both parental samples for genotyping were available in nine families. Microsatellite markers ascertained a paternal origin of the $2 q 37$ deletion in five patients and a maternal origin in four patients. We used FISH to search for the 2qtel polymorphism in eight families, and detected it in the three normal fathers of P2, P3, and P8. P2's mother was deceased. In the family of P3, the $2 \mathrm{q} 37$ deletion was of paternal origin and the father had a 2qtel polymorphism, but we could not ascertain if this polymorphic chromosome was transmitted. In the family of $\mathrm{P} 8$, the $2 \mathrm{q} 37$ deletion was of maternal origin. According to data from the fathers of P2, P3, and P8 and 14 other normal subjects (data not shown), the length of the so-called polymorphism or polymorphic 2qtel deletion was $<500 \mathrm{~kb}$. Therefore, among the parents tested, none had the same deletion as their child.

\section{Genotype-phenotype correlation}

We classified the pathological clinical features observed in the patients into three main groups: 'skeleton' for facial dysmorphism/brachydactyly, 'weight' for overweight/obesity and 'behaviour' for ASD/behavioural problems. To clarify the role of each of the deleted genes in the three groups, we listed candidate genes for these clinical features in 


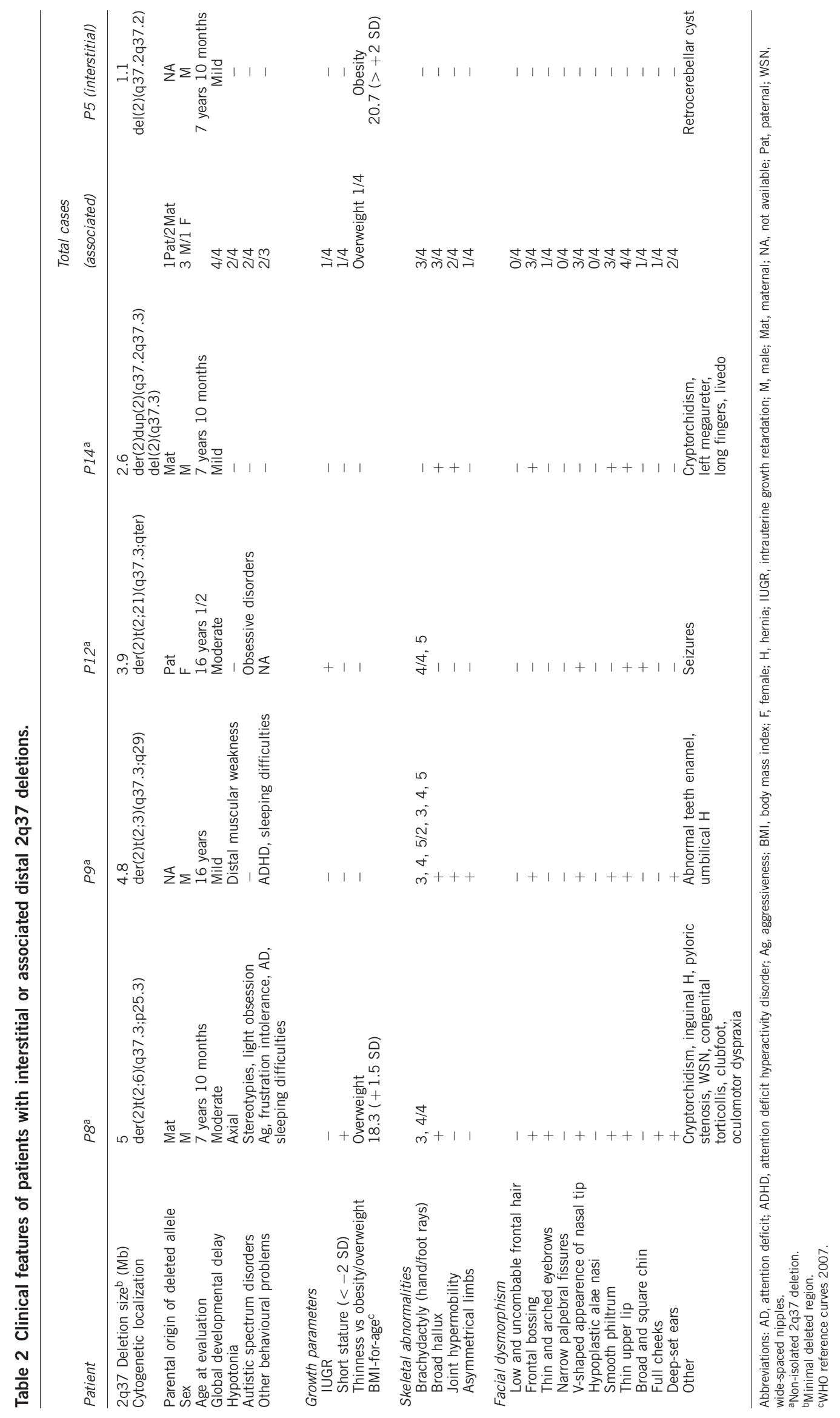



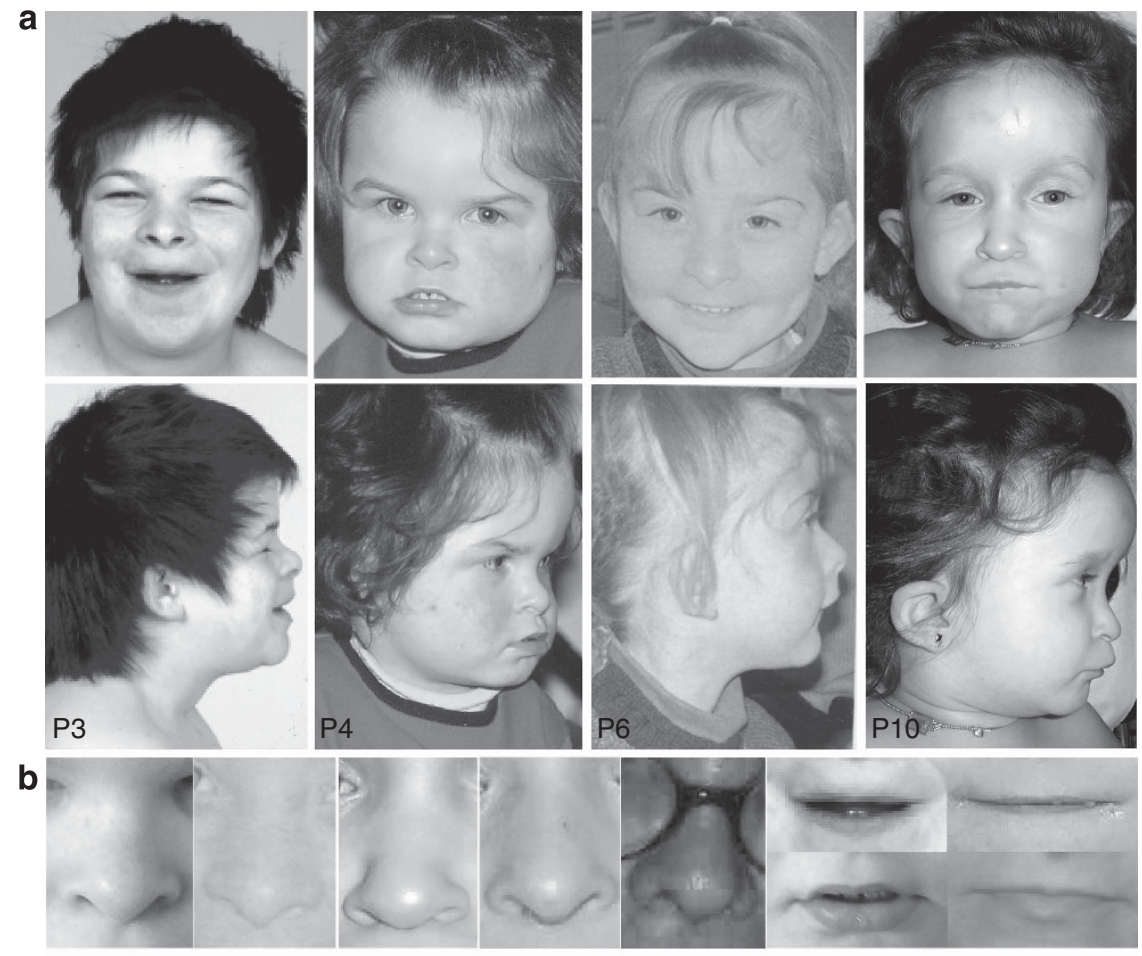

C

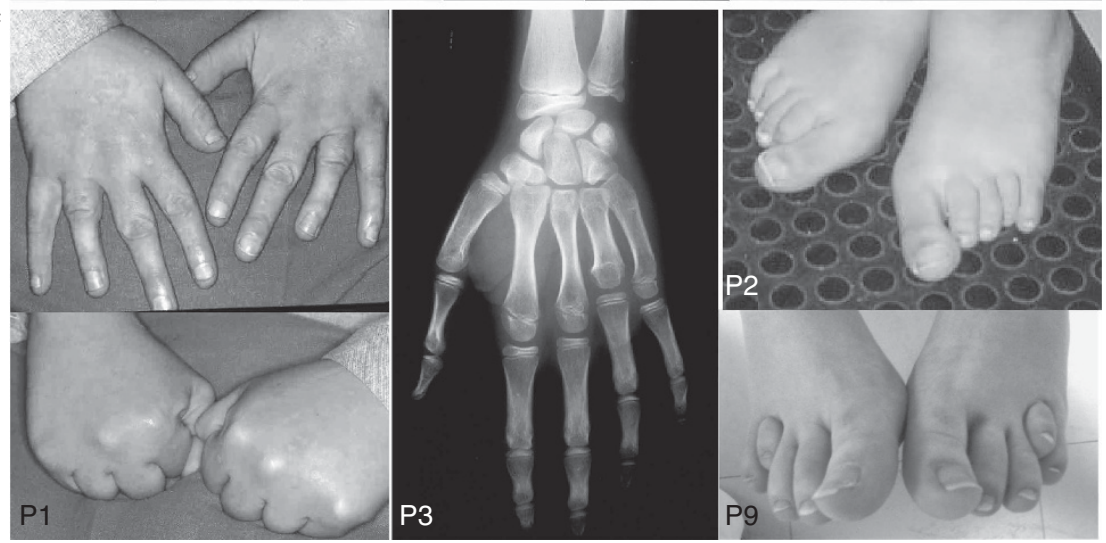

Figure 1 (a) Photographs of patients P3, P4, P6, and P10 at 17, 2, 4 and 4.5 years of age, respectively. (b) Photographs of the nose showing the V-shape of the nasal tip; photographs of the mouth showing the thin upper lip and smooth philtrum. (c) Photographs and X-rays of the hands and feet of patients P1, P3, P2 and P9, showing brachydactyly type E affecting the fourth digital ray (P1, P2 and P3) and second, third, fourth and fifth digital rays (P9).

the 14 patients and in the literature and used the Manteia database to compare them to genes from the homologous region of the mouse genome associated with a similar phenotype (Figure 3 ). To find genes related to skeletal abnormalities, we searched the database using the terms 'skeletal phenotype', 'abnormal axial skeleton morphology' and 'craniofacial phenotype'. We also included the terms 'brachydactyly' and 'abnormal paw/hand/foot morphology', however, no candidates related to these terms could be found. We searched for obesity-related genes using the term 'increased body weight' in the phenotype data set. For abnormal behaviour, we looked for genes related to 'abnormal social interaction', 'abnormal behaviour' or 'seizure' phenotypes. Two genes, PER2 (MIM 603426) and HDAC4 ${ }^{10}$ were found to be involved in all three groups. However, in this cohort, some patients without brachydactyly displayed an HDAC4 deletion, and this discrepancy needs to be explored. Thereby, to correlate the candidate genes of the Literature and Manteia with the phenotypes of our patients, we established three maps named 'skeleton', 'weight' and 'behaviour' (Figure 4).

\section{DISCUSSION}

\section{Mapping}

Subtelomeric deletions associated with developmental delays account for $2.5 \%$ of the aetiology of learning disabilities. In the largest study of the kind, in which the telomeres of 11688 individuals were investigated, the 2qtel subtelomeric deletion was a frequently encountered variation, observed in seven patients as a pure deletion. ${ }^{18}$ In the study by Ravnan et al, near half of the phenotypically altered patients had terminal $2 \mathrm{q}$ deletions, but it was not explicitly indicated whether the $2 \mathrm{q}$ deletion was a deleterious del $2 \mathrm{q} 37$ or only the common polymorphism. Indeed, the first subtelomeric FISH probes used encompassed the polymorphic loci. ${ }^{19}$ In the literature, > 115 patients with 2q37 deletions were described between 1983 and 
Table 3 2q37 Deletion mapping results and associated CNVs obtained by array-CGH (Hg 19)

\begin{tabular}{|c|c|c|c|c|c|c|}
\hline Patient & 2q Cytoband & $\begin{array}{l}\text { End of last } \\
\text { probe still } \\
\text { present (bp) }\end{array}$ & $\begin{array}{l}\text { Start of first } \\
\text { deleted probe } \\
\text { (bp) }\end{array}$ & $\begin{array}{l}\text { Minimal } \\
\text { size }(M b)\end{array}$ & Associated CNV & Comments \\
\hline P2 & q37.1q37.3 & 234436218 & 234605832 & 8.59 & No & \\
\hline P3 & q37.1q37.3 & 234655746 & 234670367 & 8.53 & No & \\
\hline P4 & q37.1q37.3 & 235022777 & 235036825 & 8.16 & No & \\
\hline P7 & q37.3 & 237985224 & 238000056 & 5.2 & No & \\
\hline P10 & q37.3 & 238798998 & 238996422 & 4.2 & No & \\
\hline P11 & q37.3 & 239084078 & 239105871 & 4.09 & $\begin{array}{l}\text { del(4)(q22.1q22.1) } \\
142 \mathrm{~kb} \\
\text { chr4:92550 144-92692347 bp }\end{array}$ & $\begin{array}{l}\text { Encompasses a partial part of FAM190A } \\
\text { (intron) }\end{array}$ \\
\hline P8 & q37.3 & 237554113 & 238258614 & 4.94 & $\begin{array}{l}\operatorname{dup}(6)(\mathrm{p} 25.3) \\
1.21 \mathrm{Mb} \\
\text { chr6:145 997-1 } 356756 \mathrm{bp}\end{array}$ & $\begin{array}{l}6 \text { p25 Duplication associated with low birth } \\
\text { weight, mental retardation, obesity, facial } \\
\text { dysmorphism and seizures } 40\end{array}$ \\
\hline P9 & q37.3 & 238410947 & 238440095 & 4.76 & $\begin{array}{l}\operatorname{dup}(3)(\mathrm{q} 29) \\
1.87 \mathrm{Mb} \\
\text { chr3:195932 835- } \\
197803820 \mathrm{bp}\end{array}$ & $\begin{array}{l}\text { 3q29 Duplication syndrome associated with } \\
\text { mental retardation, microcephaly, obesity } \\
\text { and facial dysmorphism } \\
\text { (OMIM no. 611936) }\end{array}$ \\
\hline P12 & q37.3 & 239275210 & 239307975 & 3.89 & dup(21)(qter) & $\begin{array}{l}\text { Absence of variation on chromosome } 21 \\
\text { with array-CGH }\end{array}$ \\
\hline P14 & q37.3 & 240548464 & 240575606 & 2.62 & $\begin{array}{l}\text { dup(2)(q37.2q37.3) } \\
3.51 \mathrm{Mb} \\
\text { chr2:237 } 038297- \\
240548464 \mathrm{bp}\end{array}$ & $\begin{array}{l}\text { No reported patient with pure and similar } \\
2 \text { q37 duplication and described phenotype } \\
\text { (DECIPHER and literature) }\end{array}$ \\
\hline
\end{tabular}

Abbreviations: CGH, comparative genomic hybridization; CNV, copy number variation.

2012. Among them, 74 had pure $2 q 37$ deletions (71 distal and 3 interstitial), and 41 showed associated rearrangements (37 reciprocal translocations and 4 inv/dup deletions) ${ }^{1-10,13-17,20-33}$ (Table 4). A precise mapping of the deletions, however, was carried out in only a few studies (27/115 patients). . $^{10,13,14,24,25,30,32}$ The patients with purely distal deletions (seven females, three males) included in the DECIPHER database (http://decipher.sanger.ac.uk/) had various deletion sizes ranging from 3 to $9.9 \mathrm{Mb}$. Unfortunately, clinical features were only mentioned for 2 of the 10 patients. However, clinical data were included for four females with rearranged distal deletions ranging in size from 1.59 to $5.78 \mathrm{Mb}$. In our series, we proceeded to carefully map the deletions of all patients, and observed the typical $2 \mathrm{q}$ phenotype regardless of the size of the deletion, which ranged from $8.8 \mathrm{Mb}(\mathrm{P} 1)$ to $2.6 \mathrm{Mb}(\mathrm{P} 14)$.

\section{Parental analysis}

In accordance with the report by Ravnan et al ${ }^{18}$ showing that the majority of chromosomal terminal deletions were de novo (48/60 familial studies), we confirmed in our series that, strictly speaking, no patient had inherited the $2 \mathrm{q} 37$ deletion from his/her parents. The $2 \mathrm{q}$ polymorphism is a common condition in the population $(5 \%) .^{34}$ Indeed, three parents (out of eight families tested) had the $2 \mathrm{q}$ polymorphism. Moreover, considering the possible role of the $2 \mathrm{q}$ polymorphism as a predisposing factor for the largest distal $2 \mathrm{q}$ deletion, the family of P8 illustrates the opposite trend. Indeed, the deleted $2 \mathrm{q} 37$ region was of maternal origin whereas the $2 q$ polymorphism was found in the father. Parental origin did not seem to interfere in the phenotype, as an analysis of the ratio of paternal (five) to maternal (four) origin showed no bias.

\section{Phenotype-genotype correlation}

The $2 \mathrm{q} 37$ deletion or $2 \mathrm{q}$ subtelomeric microdeletion is a condition that overlaps a recognizable '2q37-deletion syndrome' with an $\mathrm{AHO}$ like phenotype. Table 4 shows similarities between the literature and this study for clinical findings, such as developmental delays, abnormal behaviour, brachydactyly type $\mathrm{E}$ and dysmorphic features, but discrepancies can be noted with respect to weight, as discussed in a later paragraph. There was no obvious difference in phenotype 


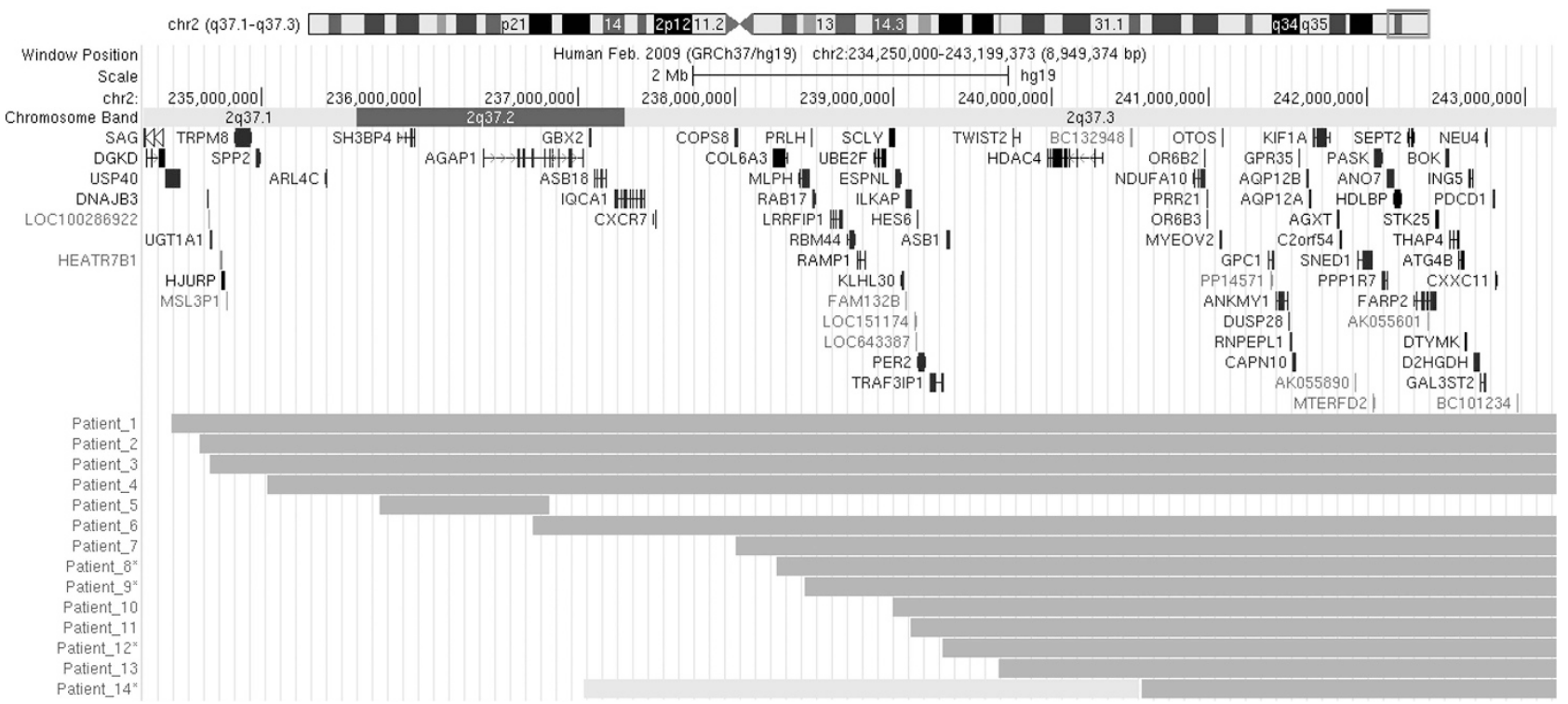

Figure 2 Map of 2q37 deletions in the 14 patients (dark grey) and of the duplicated region in P14 (light grey), with the included genes (UCSC genome browser: http://genome.ucsc.edu/cgi-bin/hgGateway).

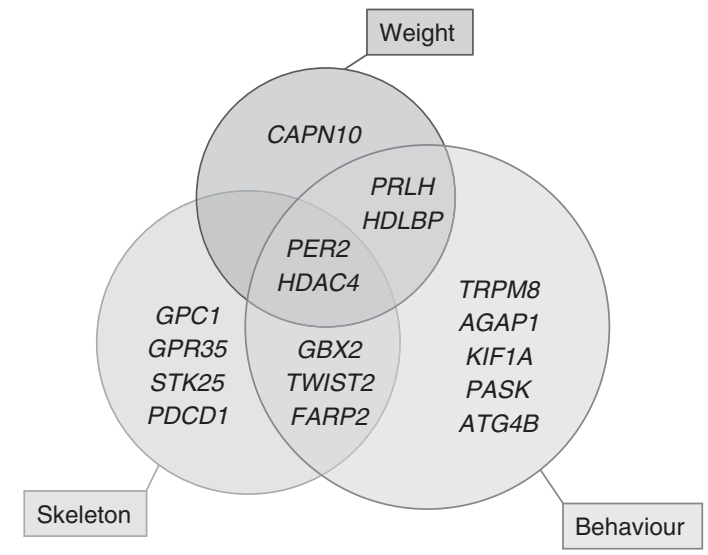

Figure 3 Graphical representation of candidate genes reported in the literature and deduced from the Manteia database for the three groups of features (skeleton, behaviour and weight).

between global (pure and associated) and pure 2q37 deletions. A sex ratio bias observed in the literature (65 females/44 males for global $2 \mathrm{q} 37$ deletions and 42 females/26 males for pure deletions) was confirmed in this study ( 8 females $/ 5$ males and 7 females $/ 2$ males, respectively; Table 4). Previously reported malformations were rare in this study and might have been coincidental, as they were more frequent when the $2 \mathrm{q} 37$ deletion was associated with another chromosomal imbalance (P8, P9, and P14; Tables 1 and 2). The cardiac malformation previously reported in $20 \%$ of patients ${ }^{12}$ was absent in the 14 patients of our cohort.

\section{Skeleton}

Among the 66 patients reviewed by Casas et al, ${ }^{23} 23 \%$ had a short stature ( $<2$ SD below the mean), as did 3 patients in this study $(21 \%$; Tables 1 and 2). Morphological dysmorphisms like brachydactyly type
$\mathrm{E}$ and facial features were the most easily recognizable symptoms of $2 q 37$ syndrome. Facial dysmorphism was present in all patients except patient P5, which could be explained by the interstitial deletion (Figure 1). The characteristic dysmorphic facial features have been previously described in detail (see for review Falk and Casas ${ }^{12}$ ) and include the shape of the nose, the appearance of the philtrum, arched eyebrows, a prominent forehead, a small mouth with thin lips and sparse hair. In our cohort, the most frequent features were the V-shaped appearance of the nasal tip, thin arched eyebrows, thin palpebral fissures, a thin upper lip with a smooth philtrum, low set ears, a large chin and hair set low on the forehead. The oldest patient (P1: 39 years) still displayed these characteristic and easily recognizable features. Candidate genes for facial dysmorphism shared by all patients and located between $240.6 \mathrm{Mb}$ (P14 breakpoint) and $242.7 \mathrm{Mb}$ at $500 \mathrm{~kb}$ from the telomeric region (Hg19) were identified as GPC1, ${ }^{15,16}$ GPR $35,{ }^{14}$ FARP2, STK $25^{11}$ and PDC1.

Brachydactyly has been previously described in about half of all patients. ${ }^{12,20,23}$ In our series, 10 of 14 patients had brachydactyly type E. The 'skeleton' map (Figure 4) is consistent with Figure 3 and shows that all patients with brachydactyly had deletions of the same 'skeleton' candidate genes such as PER2, TWIST2, HDAC4, ${ }^{10}$ GPC1, ${ }^{15,16}$ GPR 35, ${ }^{14}$ FARP2, STK25 $5^{11}$ and PDC1. HDAC4 remains the major candidate, as reported by Williams et al, ${ }^{10}$ but curiously, patients P10 and P13, who did not show brachydactyly, still had deletions of HDAC4. This could be the result of variable expressivity or an incomplete penetrance of haploinsufficiency. As a matter of fact, P13 and P10 had other skeletal disorders, such as facial dysmorphism, as well as clubfeet, sacral dimples and scoliosis in P10. The gene TWIST2 (MIM 607556) is involved in various skeletal malformations such as short limbs, abnormal vertebrae and altered craniofacial morphology in humans and rodents (OMIM, Manteia). FARP2 has been described in abnormal bone trabecular morphology related to abnormal osteoclast function (Manteia). Knockout mice for the PDCD1 gene display large spleens, lupus-like proliferative arthritis ${ }^{35}$ (Manteia), and abnormal myelopoiesis and leukopoiesis. This 


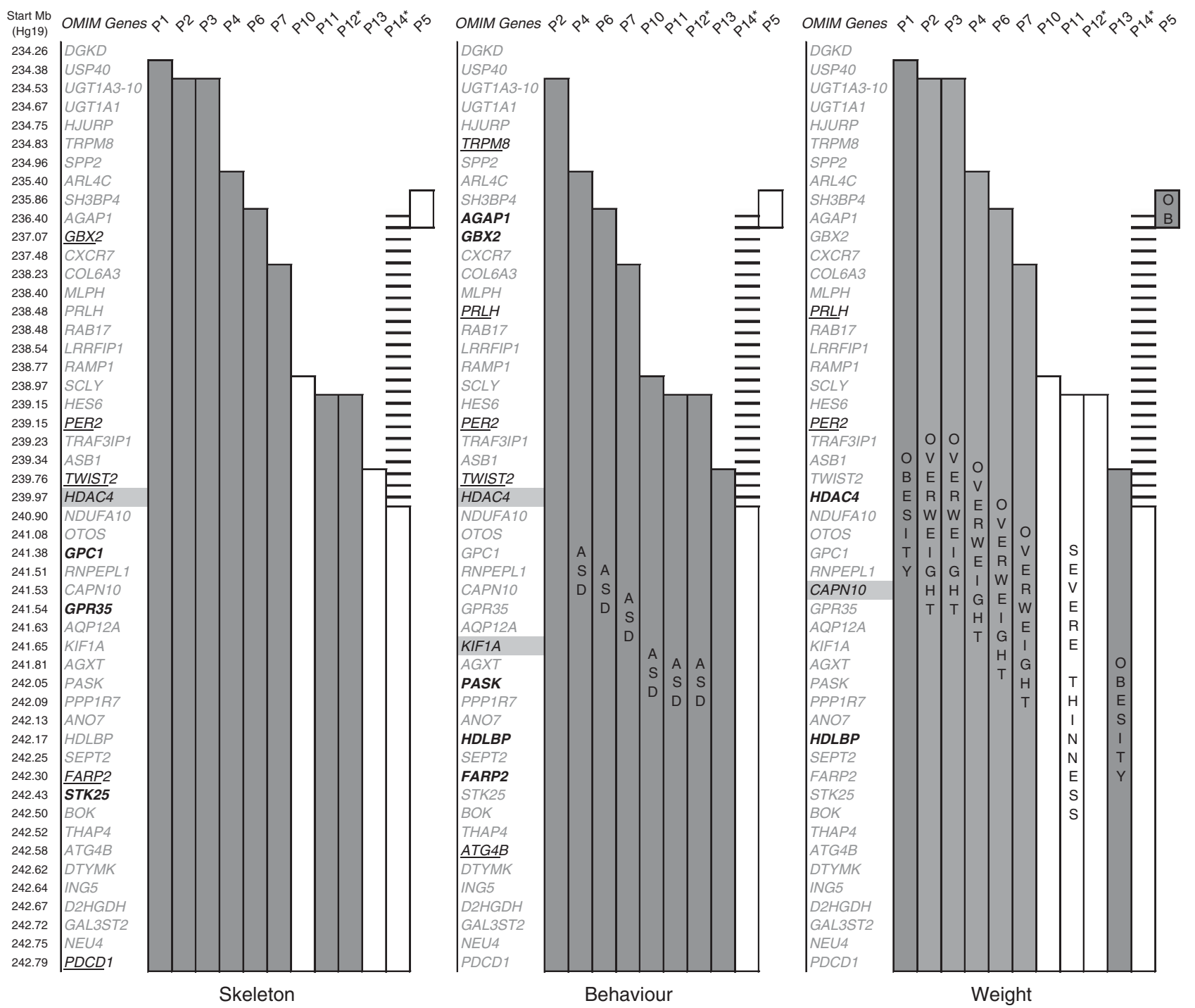

Figure 4 Representation of genotype-phenotype correlations in patients with 2q37 deletions, on three physical maps named 'skeleton', 'behaviour' and 'weight' when documented. Patients P8 and P9 were not included as their associated rearrangements may interfere in the phenotype (see Table 3). First column on the left: location of the genes ( $\mathrm{Hg} \mathrm{19).} \mathrm{On} \mathrm{each} \mathrm{of} \mathrm{the} \mathrm{three} \mathrm{maps} \mathrm{the} \mathrm{first} \mathrm{column} \mathrm{comprises} \mathrm{the} \mathrm{list} \mathrm{of} \mathrm{OMIM} \mathrm{genes} \mathrm{with} \mathrm{candidate} \mathrm{genes} \mathrm{from}$ the literature (bold), from Manteia (underlined) and both (highlighted). The columns represent the deleted genes in the patients; P5 has an interstitial deletion (last column). The columns for patients symptomatic for the concerned features are darkened. The hatched area indicates the duplicated region (P14). *Associated cytogenetic abnormality. OB, obesity.

phenotype is similar to the Felty syndrome (rheumatoid arthritis, splenomegaly and neutropenia) observed in patient P11. A mutation in the second allele of PDCD1 could explain this severe phenotype.

\section{Weight}

Among the 66 patients reviewed by Casas et al, ${ }^{23}>40 \%$ showed overweight or obesity, and a tendency toward obesity was found in older patients. In this study, overweight (6/14) was observed more frequently than obesity (3/14), mostly in patients with the largest deletions $(>4.8 \mathrm{Mb})$. The youngest patient, P6 (4 years old), was already overweight. In the literature, three genes are associated with this phenotype: $H D A C 4,{ }^{10} C A P N 10^{12}$ and HDLBP. ${ }^{12}$ Among them, CAPN10 is involved in susceptibility to human noninsulin-dependent diabetes mellitus (MIM 605286) and in increased body weight in mice (Manteia). Nevertheless, the 'overweight' map in Figure 4 shows that these three genes were also deleted in normal-weight patients (P10-12). Interestingly, the Manteia candidate gene PRLH encoding prolactin-releasing peptide (PrRP) was deleted in most overweight or obese patients (P1-4, P6 and P7) but not in patients with normal weights (P10-12 and P14). Recent research has shown the involvement of PrRP and its receptor in the control of feeding behaviour in invertebrates and vertebrates, and PrRP-deficient mice show hyperphagia. ${ }^{36,37}$ The obese patient P5 is deleted for only two genes SH3BP4 and AGAP1 (CENTG2). A patient with SH3BP4 deletion, inherited from a normal parent, is reported in the DECIPHER database (ID 254671), and four patients with a deletion interrupting AGAP1 have been described: 1 in DECIPHER (ID 251750) and three by Wassink et $a l^{17}$ but none of them were obese. Subsequently, P5 patient results may be interesting in narrowing a potential genomic region critical for obesity (AGAP1). 
Table 4 Comparison of clinical features in this study and in the literature (when documented), including 112 patients with a distal $2 q 37$ deletion $^{\mathrm{a}}$

\begin{tabular}{|c|c|c|c|c|}
\hline & \multicolumn{2}{|c|}{$\begin{array}{c}\text { Distal } 2 q 37 \text { deletions } \\
\text { global: pure and associated }\end{array}$} & \multicolumn{2}{|c|}{$\begin{array}{c}\text { Distal } 2 q 37 \text { deletions } \\
\text { pure only }\end{array}$} \\
\hline & Literature & Current study & Literature & Current study \\
\hline & 112 patients & 13 patients & 71 patients & 9 patients \\
\hline Average deletion length $(\mathrm{Mb})^{\mathrm{b}}$ & 5.13 (24 Cases) & 5.67 (13 Cases) & 5.73 (16 Cases) & 6.39 (9 Cases) \\
\hline Sex ratio & $44 \mathrm{M} / 65 \mathrm{~F}$ & $5 \mathrm{M} / 8 \mathrm{~F}$ & $26 \mathrm{M} / 42 \mathrm{~F}$ & $2 \mathrm{M} / 7 \mathrm{~F}$ \\
\hline \multirow[t]{2}{*}{ Dysmorphic features } & 103/107 & $13 / 13$ & $63 / 67$ & $9 / 9$ \\
\hline & $96.3 \%$ & $100 \%$ & $94 \%$ & $100 \%$ \\
\hline \multirow[t]{2}{*}{ Type E brachydactyly } & $60 / 78$ & $10 / 13$ & $38 / 49$ & $7 / 9$ \\
\hline & $76.9 \%$ & $76.9 \%$ & $77.6 \%$ & $77.8 \%$ \\
\hline \multirow[t]{2}{*}{ Obesity or overweight } & $28 / 69$ & $7 / 12$ & $17 / 44$ & $6 / 8$ \\
\hline & $40.6 \%$ & $58.3 \%$ & $38.6 \%$ & $75 \%$ \\
\hline \multirow[t]{2}{*}{ Developmental delay } & $106 / 106$ & $13 / 13$ & $67 / 67$ & 9/9 \\
\hline & $100 \%$ & $100 \%$ & $100 \%$ & $100 \%$ \\
\hline \multirow[t]{2}{*}{ Abnormal behaviour } & $47 / 54$ & $10 / 11$ & $32 / 38$ & $7 / 7$ \\
\hline & $87 \%$ & $90.9 \%$ & $84.2 \%$ & $100 \%$ \\
\hline \multirow[t]{2}{*}{ Autistic spectrum disorders } & $32 / 49$ & $7 / 11$ & $25 / 39$ & $5 / 7$ \\
\hline & $65.3 \%$ & $63.6 \%$ & $64.1 \%$ & $71.4 \%$ \\
\hline
\end{tabular}

Abbreviations: $F$, female; $M$, male.

aFor review see Falk and Casas ${ }^{12}$ and Casas et al. ${ }^{23}$

bWhen documented.

In Table 4, the discrepancy between pure $2 \mathrm{q} 37$ deletions in the literature and in this study ( $38.6 \%$ vs $75 \%$ ) could be explained by the lack of availability of BMI curves for previous reports, and semantic heterogeneity (overweight $v s$ obesity). Indeed, the BMI-for-age has to be established to determine weight gain, and these curves were not available for most reported children.

\section{Intellectual deficiency and seizures}

Among previously reported patients for whom clinical evaluations were carried out, $100 \%$ had either a global developmental delay or hypotonia, and mild to severe mental retardation. In this series, five patients had a history of hypotonia, all patients had intellectual deficiency with mild to severe developmental delays but without major motor deficits, and P10 and P11 showed no language acquisition. Seizures have been previously described in $20-35 \%$ of patients with $2 \mathrm{q} 37$ deletions $\mathrm{s}^{20,38}$ and were present here in 3 patients: P11 and P12 had generalized seizures and P6 had febrile seizures. The candidate genes for seizures identified using Manteia were HDAC4 and $\mathrm{D} 2 \mathrm{HGDH}$. Both were deleted in P6, P11 and P12. Interestingly, Williams et al have described an epileptic patient (2282) with a deletion distal to HDAC4, including D2HGDH. ${ }^{10} \mathrm{D} 2 \mathrm{HGDH}$ is involved in D-2-hydroxyglutaric aciduria (MIM 600721), a neurometabolic disorder characterized by developmental delays, epilepsy, hypotonia and dysmorphic features.

\section{Behavioural disorders}

As shown by Falk and Casas ${ }^{12}$, multiple reports have documented a behavioural phenotype overlapping the autistic spectrum. A diagnosis of autism or description of autistic behaviour has been reported in $24-35 \%$ of patients. ${ }^{20,23}$ Most of the patients in our study had heterogeneous behavioural disorders, with seven in the autistic spectrum, and patients $\mathrm{P} 4$ and P11 were reported as being autistic. The 'behaviour' map shows that all affected patients shared a deletion of the following candidate genes: TWIST2, HDAC4, ${ }^{10} \mathrm{KIF} 1 \mathrm{~A},{ }^{15}$ PASK, ${ }^{5}$ HDLBP, ${ }^{5}$ FARP $2,{ }^{5}$ and ATG4B. P14 (del/dup 2q37.3) showed normal behaviour but carried deletions of the same candidate genes, except for HDAC4 and TWIST2. HDAC4 mutations and deletions have been reported as being associated with self-injurious and aggressive behaviour. ${ }^{10}$ However, other genes could be involved, as evoked by the deletion distal to HDAC4 in the autistic patient referred to above (2282). ${ }^{10}$ TWIST2 has been reported to be involved in knockout mice with dystonic movements. ${ }^{39}$ $A G A P 1^{13,17}$ is another candidate gene located in $2 \mathrm{q} 37$ and the four patients previously cited with AGAP1 deletion (ID 251750 and Wassink et $a l^{17}$ ) had autistic disorders. Interestingly, P5 had a similar deletion but no behavioural problems. KIF1A, highlighted by the literature ${ }^{15}$ and Manteia, encodes a motor protein involved in the anterograde transport of synaptic-vesicle precursors along axons, and a mutation in this gene has been reported in a patient with nonsyndromic intellectual disability (MIM 601255). KIF1A is also associated with hypoactivity and hyporesponse to tactile stimuli in knockout mice (Manteia).

In data from Manteia, the gene PER2 was shared by the three phenotypic groups (Figure 3). A single reference in humans has reported a heterozygous mutation in PER2 in advanced sleep-phase syndrome. Interestingly, PER2 is a member of the period family of genes, associated in $\mathrm{KO}$ mice with abnormal food intake, sleep patterns, social interaction, body weight gain and skeletal development.

\section{CONCLUSION}

From these 14 new patients with a $2 \mathrm{q} 37$ or subtelomeric deletion, we mapped the smallest region of overlap and the genes responsible for facial dysmorphism, brachydactyly, overweight and behavioural problems. The $2 \mathrm{q} 37$ subtelomeric region is a gene-rich region spanning $8.8 \mathrm{Mb}$ for our largest deletion (Figure 2). The $2.6 \mathrm{Mb}$ region of overlap contains $>25$ OMIM genes. Of these, some, such as HDAC4, are known to be implicated in behavioural disorders, autism or intellectual disability. The molecular data from our group of patients added to observations from the literature and the DECIPHER database allowed us to focus on other candidate genes. This deletion is still under-diagnosed, but the presence of brachydactyly 
type $\mathrm{E}$ and the characteristic facial features could direct clinicians toward this syndrome. However, a few patients with pure deletions did not display the most recognizable features (eg, P10 and P13 missed brachydactyly), or possessed a more severe phenotype (eg, P4 had severe behavioural trouble and P11 displayed Felty's syndrome). This could be explained by variations in genetic background, mutations in the second allele or epigenetic phenomena, such as imprinting, although the sex ratio of the inherited deletion is not in favour of the last hypothesis. The expression of regulatory elements in the deleted region or at distance upstream or downstream may also interfere with the phenotype of the $2 \mathrm{q} 37$ subtelomeric microdeletion. The associated copy number variations have been explored in the present patients and no potential modifier emerged (Table 3). ${ }^{40}$ Furthermore, genetic, as well as functional, analysis would be useful as deletion of HDAC4 was reported as resulting in reducing expression of RAI1. ${ }^{10}$ These studies may provide new insights into the pathogenic role of the haploinsufficient 2q37 genes.

\section{CONFLICT OF INTEREST}

The authors declare no conflict of interest.

\section{ACKNOWLEDGEMENTS}

We thank the patients and their families. We thank our colleagues in the ACLF network 'mapping the subtelomeric regions/clinical and molecular phenotype' This work was funded by the following grants: AOL 2001 and AOL 2003, PHRC 2003 CHU REIMS, DHOS/OPRC/2007/35 and 455 France. The manuscript was prepared with the editorial help from Gap Junction.

1 Balikova I, Vermeesch JR, Fryns JP, Van Esch H: Bronchiectasis and immune deficiency in an adult patient with deletion 2 q37 due to an unbalanced translocation $\mathrm{t}(2 ; 10)$. Eur J Med Genet 2009; 52: 260-261.

2 Chen CP, Lin SP, Chern SR et al: Deletion 2q37.3-> qter and duplication 15q24.3$>$ qter characterized by array CGH in a girl with epilepsy and dysmorphic features. Genet Couns 2010; 21: 263-267.

3 Cusco I, del Campo M, Vilardell $\mathrm{M}$ et al: Array-CGH in patients with Kabuki-like phenotype: identification of two patients with complex rearrangements including 2q37 deletions and no other recurrent aberration. BMC Med Genet 2008; 9: 27.

4 Devillard F, Guinchat V, Moreno-De-Luca D et al: Paracentric inversion of chromosome 2 associated with cryptic duplication of $2 \mathrm{q} 14$ and deletion of $2 \mathrm{q} 37$ in a patient with autism. Am J Med Genet A 2010; 152A: 2346-2354.

5 Felder B, Radlwimmer B, Benner A et al: FARP2, HDLBP and PASK are downregulated in a patient with autism and 2q37.3 deletion syndrome. Am J Med Genet A 2009; 149A: 952-959.

6 Kariminejad A, Kariminejad R, Tzschach A et al: Craniosynostosis in a patient with 2q37.3 deletion 5q34 duplication: association of extra copy of MSX2 with craniosynostosis, Am J Med Genet A 2009; 149A: 1544-1549.

7 Kitsiou-Tzeli S, Sismani C, loannides M et al: Array-CGH analysis and clinical description of 2q37.3 de novo subtelomeric deletion. Eur J Med Genet 2007; 50 $73-78$

8 Mazzone L, Vassena L, Ruta L, Mugno D, Galesi O, Fichera M: Brief report: peculiar evolution of autistic behaviors in two unrelated children with brachidactyly-menta retardation syndrome. J Autism Dev Disord 2012; 42: 2202-2207.

9 Vera-Carbonell A, Lopez-Exposito I, Bafalliu JA et al: Molecular characterization of a new patient with a non-recurrent inv dup del $2 q$ and review of the mechanisms for this rearrangement. Am J Med Genet A 2010; 152A: 2670-2680.

10 Williams SR, Aldred MA, Der Kaloustian VM et al: Haploinsufficiency of HDAC4 causes brachydactyly mental retardation syndrome, with brachydactyly type $\mathrm{E}$, developmental delays, and behavioral problems. Am J Hum Genet 2010; 87: 219-228.

11 Davids MS, Crawford E, Weremowicz S et al: STK25 is a candidate gene for pseudopseudohypoparathyroidism. Genomics 2001; 77: 2-4.

12 Falk RE, Casas KA: Chromosome 2q37 deletion: clinical and molecular aspects. Am J Med Genet C Semin Med Genet 2007; 145C: 357-371.

13 Lukusa T, Vermeesch JR, Holvoet M, Fryns JP, Devriendt K: Deletion 2q37.3 and autism: molecular cytogenetic mapping of the candidate region for autistic disorder. Genet Couns 2004; 15: 293-301.
14 Shrimpton AE, Braddock BR, Thomson LL, Stein CK, Hoo JJ: Molecular delineation of deletions on 2 q37.3 in three cases with an Albright hereditary osteodystrophy-like phenotype. Clin Genet 2004; 66: 537-544.

15 Smith M, Escamilla JR, Filipek $P$ et al: Molecular genetic delineation of 2q37.3 deletion in autism and osteodystrophy: report of a case and of new markers for deletion screening by PCR. Cytogenet Cell Genet 2001; 94: 15-22.

16 Syrrou M, Keymolen K, Devriendt $\mathrm{K}$ et al: Glypican 1 gene: good candidate for brachydactyly type E. Am J Med Genet 2002; 108: 310-314.

17 Wassink TH, Piven J, Vieland VJ et al: Evaluation of the chromosome 2q37.3 gene CENTG2 as an autism susceptibility gene. Am J Med Genet B Neuropsychiatr Genet 2005; 136B: 36-44.

18 Ravnan JB, Tepperberg JH, Papenhausen P et al: Subtelomere FISH analysis of 11 688 cases: an evaluation of the frequency and pattern of subtelomere rearrangements in individuals with developmental disabilities. J Med Genet 2006; 43: 478-489.

19 Knight SJ, Lese CM, Precht KS et al: An optimized set of human telomere clones for studying telomere integrity and architecture. Am J Hum Genet 2000; 67: 320-332.

20 Aldred MA, Sanford RO, Thomas NS et al: Molecular analysis of 20 patients with 2q37.3 monosomy: definition of minimum deletion intervals for key phenotypes. J Med Genet 2004; 41: 433-439.

21 Armstrong L, Allanson JE, Weaver DD, Bevan CJ, Hobart $\mathrm{HH}$ : Unrelated patients with a rearrangement of chromosome 2 causing duplication of 2 p23 and deletion of $2 q 37$. Am J Med Genet A 2005; 134: 299-304.

22 Burd L, Martsolf JT, Kerbeshian J, Jalal SM: Partial 6p trisomy associated with infantile autism. Clin Genet 1988; 33: 356-359.

23 Casas KA, Mononen TK, Mikail CN et al: Chromosome 2q terminal deletion: report of 6 new patients and review of phenotype-breakpoint correlations in 66 individuals. Am J Med Genet A 2004; 130A: 331-339.

24 Chaabouni M, Le Merrer M, Raoul 0 et al: Molecular cytogenetic analysis of five 2q37 deletions: refining the brachydactyly candidate region. Eur J Med Genet 2006; 49 255-263.

25 Chassaing N, De Mas P, Tauber M et al: Molecular characterization of a cryptic 2q37 deletion in a patient with Albright hereditary osteodystrophy-like phenotype. Am J Med Genet A 2004; 128A: 410-413.

26 Fernandez-Rebollo E, Perez O, Martinez-Bouzas C et al: Two cases of deletion 2q37 associated with segregation of an unbalanced translocation 2;21: choanal atresia leading to misdiagnosis of CHARGE syndrome. Eur J Endocrinol 2009; 160 711-717.

27 Galasso C, Lo-Castro A, Lalli C, Nardone AM, Gullotta F, Curatolo P: Deletion 2q37: an identifiable clinical syndrome with mental retardation and autism. J Child Neurol 2008; 23: 802-806.

28 Giardino D, Finelli P, Gottardi G et al: Cryptic subtelomeric translocation $\mathrm{t}(2 ; 16)(\mathrm{q} 37 ; \mathrm{q} 24)$ segregating in a family with unexplained stillbirths and a dysmorphic, slightly retarded child. Eur J Hum Genet 2001; 9: 881-886.

29 Grammatico P Majore $S$, Marrocco G et al: 46,XX,der(2)t(2;10) (2pter->2q37::10p13->10pter)[127]/45,X,der(2)t(2;10) (2pter->2q37::10p13$>10$ pter)[23]. Karyotype-phenotype correlation and genetic counselling in complex karyotypes. Genet Couns 1999; 10: 351-358.

30 Lukusa T, Smeets E, Vogels A, Vermeesch JR, Fryns JP: Terminal 2q37 deletion and autistic behaviour. Genet Couns 2005; 16: 179-180.

31 Sanchez JM, Pantano AM: A case of deletion 2q35 - qter and a peculiar phenotype. $J$ Med Genet 1984; 21: 147-149.

32 Sogaard M, Tumer Z, Hjalgrim H et al: Subtelomeric study of 132 patients with mental retardation reveals 9 chromosomal anomalies and contributes to the delineation of submicroscopic deletions of 1pter, 2qter, 4pter, 5qter and 9qter. BMC Med Genet 2005; 6: 21

33 Wiktor A, Feldman GL, Bawle EV, Czarnecki P, Conard JV, Van Dyke DL: Deletion of 2q37 and duplication of 10q24: two cases in the same family and review of the literature. Ann Genet 2001; 44: 129-134.

34 Fan YS, Zhang Y, Speevak M, Farrell S, Jung JH, Siu VM: Detection of submicroscopic aberrations in patients with unexplained mental retardation by fluorescence in situ hybridization using multiple subtelomeric probes. Genet Med 2001; 3: 416-421.

35 Nishimura H, Nose M, Hiai H, Minato N, Honjo T: Development of lupus-like autoimmune diseases by disruption of the PD- 1 gene encoding an ITIM motif-carrying immunoreceptor. Immunity 1999; 11: 141-151.

36 Maletinska L, Spolcova A, Maixnerova J, Blechova M, Zelezna B: Biological properties of prolactin-releasing peptide analogs with a modified aromatic ring of a C-terminal phenylalanine amide. Peptides 2011; 32: 1887-1892.

37 Takayanagi Y, Onaka T: Roles of prolactin-releasing peptide and RFamide related peptides in the control of stress and food intake. FEBS J 2010; 277: 4998-5005.

38 Doherty ES, Solomon BD, Lacbawan F: 2 q37 Deletion Syndrome; in Pagon RA Bird TD, Dolan CR, Stephens K (Hrsg) (eds): Gene Reviews. Seattle/WA: University of Washington, 1993.

39 Sosic D, Richardson JA, Yu K, Ornitz DM, Olson EN: Twist regulates cytokine gene expression through a negative feedback loop that represses NF-kappaB activity. Cell 2003; 112: 169-180.

40 Vermeesch JR, Thoelen R, Fryns JP: A familial complex chromosome translocation resulting in duplication of 6p25. Ann Genet 2004; 47: 275-280. 